\title{
The predictive utility of patient-reported outcomes and performance status for survival in metastatic lung cancer patients treated with chemoimmunotherapy
}

\author{
Sarah Badaoui ${ }^{1 \#}$, Adel Shahnam ${ }^{2 \#}$, Ross A. McKinnon ${ }^{1}$, Ahmad Y. Abuhelwa ${ }^{1}$, Michael J. Sorich $^{1 *}$, \\ Ashley M. Hopkins ${ }^{1 *}$ \\ ${ }^{1}$ College of Medicine and Public Health, Flinders University, Adelaide, Australia; ${ }^{2}$ Crown Princess Mary Cancer Center, Westmead Hospital, Sydney, \\ New South Wales, Australia \\ Contributions: (I) Conception and design: S Badaoui, A Shahnam, MJ Sorich, AM Hopkins; (II) Administrative support: A Shahnam, RA McKinnon, \\ MJ Sorich, AM Hopkins; (III) Provision of study materials or patients: AM Hopkins, AY Abuhelwa, MJ Sorich; (IV) Collection and assembly of data: \\ S Badaoui, AM Hopkins, AY Abuhelwa, MJ Sorich; (V) Data analysis and interpretation: S Badaoui, A Shahnam, RA McKinnon, AY Abuhelwa, MJ \\ Sorich, AM Hopkins; (VI) Manuscript writing: All authors; (VII) Final approval of manuscript: All authors. \\ \#These authors contributed equally to this work. \\ *These authors contributed equally to this work. \\ Correspondence to: Professor Michael J. Sorich. Room 5E315, Flinders Medical Centre, Bedford Park, Adelaide, South Australia 5042, Australia. \\ Email: michael.sorich@flinders.edu.au.
}

Background: Atezolizumab, an immune checkpoint inhibitor, in combination with chemotherapy (chemoimmunotherapy) has become a first-line treatment option for metastatic non-small cell lung cancer (NSCLC). Patient-reported outcomes (PROs) are self-reported measures that have shown promise in their predictive value for survival. However, there have been no studies that have assessed the prognostic performance of PROs in an advanced NSCLC cohort initiating first-line atezolizumab based chemoimmunotherapy.

Methods: This study used individual-participant data (IPD) from the IMpower130, IMpower131 and IMpower150 clinical trials. Cox proportional hazards regression was utilized to determine the association between pre-treatment PROs with overall survival (OS) and progression free survival (PFS). The prediction performance of PROs was assessed using the C-statistic. For the PRO measure identified as the most predictive of survival, an exploratory analysis comparing the predictive performance against Eastern Cooperative Oncology Group Performance Status (ECOG-PS) was conducted.

Results: Patient-reported physical function, fatigue, appetite loss, pain, role function, global health status, social function, dyspnoea, constipation, nausea and vomiting, insomnia, emotional function, cognitive function, and financial difficulty were statistically associated with $\mathrm{OS}(\mathrm{P}<0.05)$. Physical function $(\mathrm{c}=0.62)$, fatigue $(c=0.61)$, and appetite loss $(c=0.60)$ were the most predictive variables for OS. Patient-reported physical function ( $\mathrm{c}=0.60$ ) also had higher predictive performance than physician-defined ECOG-PS (c=0.57). Conclusions: In patients with advanced NSCLC who received first line atezolizumab based therapy, pre-treatment PROs were prognostic for survival outcomes. Patient-reported physical function had higher predictive performance compared to physician-defined ECOG-PS. These results suggest PROs have significant worth in clinical practice and research trials of ICIs as a stratification factors.

Keywords: Patient-reported outcomes; prognosis; survival; immunotherapy; non-small cell lung cancer (NSCLC)

$\wedge$ ORCID: 0000-0002-0043-8374. 
Submitted Nov 26, 2021. Accepted for publication Feb 12, 2022.

doi: $10.21037 /$ tlcr-21-938

View this article at: https://dx.doi.org/10.21037/tlcr-21-938

\section{Introduction}

The combination of immune checkpoint inhibitors (ICIs) with traditional chemotherapies (chemoimmunotherapy) has recently been established as a frontline treatment mainstay for advanced non-small cell lung cancer (NSCLC) (1). Coincidingly, there is a paucity of evidence on the prognostic value of clinicopathological data with chemoimmunotherapy, which is limiting informed decision making by oncologists and patients.

Patient-reported outcomes (PROs) are easily collected self-reported measures of physical, emotional, social, cognitive, and disease status, and they are commonly used in oncology trials and clinical practice to evaluate the quality of life of patients with cancer undergoing treatment $(2,3)$. Serially collected PROs provide a multifaceted snapshot of patient-centered data at multiple timepoints $(4,5)$. In addition, several recent studies highlight pre-treatment PROs are predictive of likely survival. For example, patientreported physical function has been shown a tumoragnostic predictor of survival, which in several analyses has been more predictive than physician-assessed Eastern Cooperative Oncology Group performance status (ECOGPS) (6-11). While this indicates that PROs may have a role in informing clinical trial design and better aiding shared decision making, there have been no studies that have assessed the prognostic performance of PROs in an advanced NSCLC cohort treated with emerging first-line chemoimmunotherapy approaches.

This study aimed to evaluate the prognostic performance of pre-treatment PROs and ECOG-PS in patients with advanced NSCLC initiating first-line atezolizumabchemotherapy approaches. We present the following article in accordance with the REMARK reporting checklist (available at https://tlcr.amegroups.com/article/ view/10.21037/tlcr-21-938/rc).

\section{Methods}

\section{Inclusion criteria and trial description}

This study utilized deidentified individual-participant data (IPD) from phase 3 clinical trials IMpower130 (NCT02367781), IMpower131 (NCT02367794), and
IMpower150 (NCT02366143) (12-14). The inclusion criteria have previously been described (12-14). All trials included patients above the age of 18 with a baseline ECOG-PS of 0 or 1 (12-14). IMpower130 investigated the combination of atezolizumab and chemotherapy (carboplatin plus nab-paclitaxel), versus chemotherapy alone and included patients with stage IV non-squamous-NSCLC who had no prior chemotherapy treatment or were intolerant to at least one tyrosine kinase inhibitor (13). IMpower131 investigated $(1: 1: 1)$ the combination of atezolizumab plus carboplatin plus paclitaxel, versus atezolizumab plus carboplatin plus nab-paclitaxel, versus carboplatin plus nab-paclitaxel (12). IMpower131 included only stage IV squamous NSCLC (12). IMpower150 investigated (1:1:1) the combination of atezolizumab plus carboplatin plus paclitaxel, versus atezolizumab plus bevacizumab plus carboplatin plus paclitaxel, versus carboplatin plus paclitaxel (14). IMpower150 only included patients with stage IV non-squamous-NSCLC (14).

\section{Predictor and outcome definitions}

The primary outcome of this study was overall survival (OS), with progression free survival (PFS) assessed as a secondary outcome. In the IMpower130, IMpower131 and IMpower150 clinical trials, PFS and OS were measured from randomization to first documented disease progression using RECIST version 1.1 and death from any cause, respectively (12-14).

PROs measures were recorded in all 3 clinical trials using the European Organisation for Research and Treatment of Cancer (EORTC) QLQ-C30 (version 3.0) questionnaire $(2,15,16)$. The EORTC QLQ-C30 is comprised of 30 questions capturing answers ranging from 1 ("Not at all") to 4 ("Very much"). The questionnaire answers are convertible to five functional scales (physical, role, cognitive, emotional, and social), nine symptom scales (fatigue, pain, nausea and vomiting, dyspnea, loss of appetite, insomnia, constipation, diarrhea, and financial difficulties) and a global health scale $(2,16)$. These scales are validated measures which range between 0 and 100. For functional scale measures, higher scores represent a better level of function from a patient perspective. For symptom scales, higher scores represent a 
worse level of symptoms from a patient perspective (17).

\section{Statistical analysis}

The association between pre-treatment PROs with OS and PFS were assessed via Cox proportional hazards regression. Results were reported as hazard ratios (HR) with $95 \%$ confidence intervals $(95 \%$ CI). Statistical significance was pre-defined at $\mathrm{P}$ value $<0.05$. Complete case analyses were conducted. The prediction performance of PROs was assessed using the C-statistic (c). Akaike information criterion (AIC) and visual checks were used to assess potential non-linearity and model fit. Analyses were stratified by study and treatment. Analyses were adjusted for baseline age, sex, race, ECOG-PS, smoking history, histology, liver metastasis, PD-L1 and comorbidity count.

Kaplan-Meier analysis was used to graphically represent the association between pre-treatment PROs with survival outcomes. For graphical plots, PROs were grouped into tertiles (low, intermediate, high scores) using the EORTC QLQ-C30 Tables of Reference Values for the "Lung Module: Stage III/IV" cohort (18). "Low" represents PROs scores within the lower 25 th percentile of the reference group, "intermediate" within the middle 50th percentiles, and "high" within the upper 25th percentile.

For the PROs measure identified as the most predictive of survival in the pooled cohort, an exploratory analysis comparing the predictive performance against ECOG-PS was conducted.

All analyses were performed using $\mathrm{R}$ version 3.6.2.

\section{Ethical consideration}

The study was conducted in accordance with the Declaration of Helsinki (as revised in 2013). Secondary analysis of anonymized clinical trial data was confirmed negligible risk research by the Southern Adelaide Local Health Network, Office for Research and Ethics and was exempt from review as the project utilised publicly available de-identified data.

\section{Results}

\section{Patient population}

In the pooled cohort, 1,932 patients were treated with atezolizumab based chemoimmunotherapy and 960 patients were treated with chemotherapy without atezolizumab.
Table S1 summarizes the baseline patient characteristics by treatment arms. Differences in distribution by treatment arm were observed with respect to age, sex, race, smoking history, histology and comorbidity count. Except for patient-reported dyspnoea and constipation, the distributions of pre-treatment PROs values were similar between study cohorts (Table S2). Median follow-up was 18 months in the pooled sample.

\section{Prognostic associations of PROs}

In the pooled cohort, on both univariable and adjusted analysis, patient-reported physical function, fatigue, appetite loss, pain, role function, global health status, social function, dyspnoea, constipation, nausea and vomiting, insomnia, emotional function, cognitive function, and financial difficulty were statistically associated with OS $(\mathrm{P}<0.05$; Table S3). Patient reported physical function $(\mathrm{c}=0.62)$, fatigue $(\mathrm{c}=0.61)$, and appetite loss $(\mathrm{c}=0.60)$ were the most predictive variables for OS. The prognostic significance of patient-reported physical function, fatigue, and appetite loss was consistent across study cohorts (Figure S1). Similar findings were seen with PFS, with the exception that financial difficulty was not statistically significant on adjusted analysis (Table S4). Figure 1 illustrates KaplanMeier estimates of OS by patient-reported physical function, stratified by study and treatment arm for patients treated with atezolizumab-based chemoimmunotherapy.

\section{Comparison of patient-reported physical function with ECOG-PS}

With patient-reported physical function being the most prognostic PROs in the study cohort, the study went on to perform an exploratory analysis comparing its predictive performance against physician-defined ECOG-PS.

In the cohort of 1,927 participants, 191 (10\%) had missing pre-treatment patient-reported physical function values (Table S5). In the evaluable cohort, 270 (14\%) patients self-reported low physical function, 1,010 (52\%) self-reported intermediate physical function and 456 (24\%) self-reported high physical function. In the evaluable cohort, 761 patients were assigned an ECOG-PS of 0 , and 1,166 an ECOG-PS of 1 (Table S5). On univariable analysis, the OS prediction performance (c) of ECOG-PS $(1+v s .0)$ was $0.57(\mathrm{P}<0.001 ;$ Table 1). Comparatively, the OS prediction performance of the patient-reported physical function groups were slightly higher at $0.60(\mathrm{P}<0.001$; Table 1$)$. 

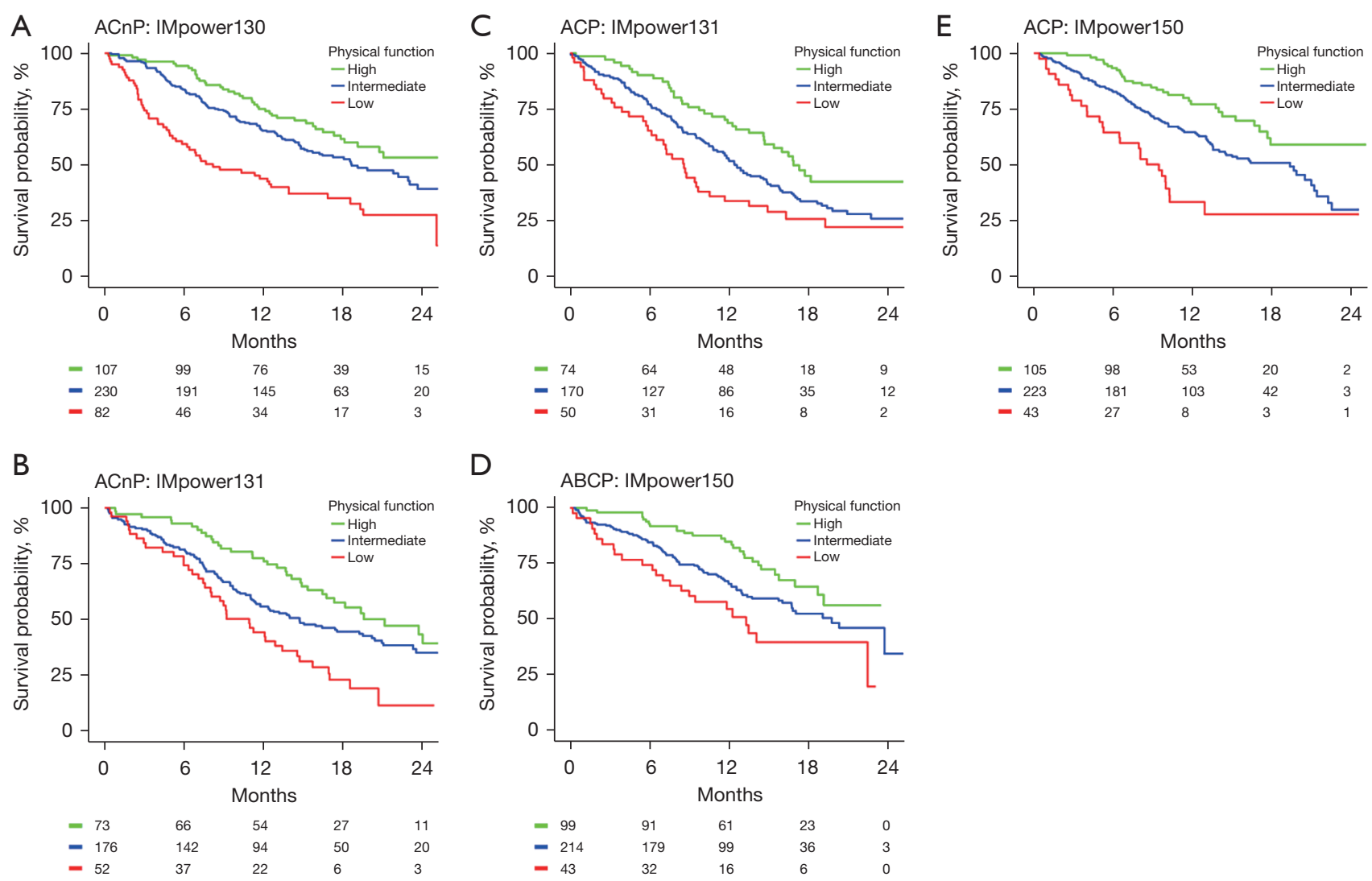

Figure 1 Kaplan-Meier estimates of OS by patient-reported physical function for patients treated with atezolizumab. ACnP, atezolizumab plus carboplatin plus nab-paclitaxel; ACP, atezolizumab plus carboplatin plus paclitaxel; ABCP, atezolizumab plus bevacizumab plus carboplatin plus paclitaxel.

Table 1 Associations between patient-reported physical function and ECOG-PS with OS for patients treated with atezolizumab

\begin{tabular}{|c|c|c|c|c|c|c|c|}
\hline \multirow{2}{*}{ Parameter } & \multicolumn{4}{|c|}{ Univariable } & \multicolumn{3}{|c|}{ Multivariable } \\
\hline & $\mathrm{n}$ & $\mathrm{HR}[95 \% \mathrm{Cl}]$ & $\mathrm{P}$ & c & $\mathrm{n}$ & $\mathrm{HR}[95 \% \mathrm{Cl}]$ & $\mathrm{P}$ \\
\hline Physical function & & & $<0.001$ & 0.60 & & & $<0.001$ \\
\hline Low & 270 & 2.75 [2.23 to 3.40$]$ & & & 270 & 2.38 [1.92 to 2.95$]$ & \\
\hline Intermediate & 1,013 & 1.59 [1.33 to 1.89$]$ & & & 1,010 & 1.49 [1.25 to 1.78$]$ & \\
\hline ECOG-PS & & & $<0.001$ & 0.57 & & & $<0.001$ \\
\hline 0 & 761 & 1 & & & 671 & & \\
\hline $1+$ & 1,166 & $1.72[1.50$ to 1.97$]$ & & & 1,065 & 1.53 [1.32 to 1.78$]$ & \\
\hline
\end{tabular}

\#, model includes pre-treatment physical function and ECOG-PS. Cl, confidence interval; c, c-statistic; ECOG-PS, Eastern Cooperative Oncology Group Performance Status; HR, hazard ratio; n, number. 
Demonstrating independent prognostic information, in a multivariable model including both patient-reported physical function and physician-assessed ECOG-PS, the OS prediction performance increased to 0.62 with both variables showing statistical significance $(\mathrm{P}<0.001$, Table 1$)$. Sensitivity analysis indicated that on univariable analysis, the PFS prediction performance of the patient-reported physical function groups was also slightly higher; more importantly, multivariable analysis demonstrated both variables provide independent prognostic information $(\mathrm{P}<0.001$, Table $\mathrm{S} 6)$.

Notably, of the 761 patients with a clinician defined ECOG-PS of 0 (fully active, without restriction), 424 (56\%) self-reported low or intermediate physical function scores, $140(18 \%)$ reported having 'quite a bit' or 'very much' trouble doing strenuous activities and 152 (20\%) reported having 'quite a bit' or 'very much' trouble taking a long walk (Table S5)-highlighting a discordance between clinician defined ECOG-PS score of 0 and patient-reported physical function. Similar results were observed for PFS $(\mathrm{P}<0.001$, Table S6).

\section{Discussion}

This study showed the pre-treatment PROs were independent prognostic factors for survival outcomes in advanced NSCLC patients treated with first-line atezolizumab combination therapies. Of which the most predictive for OS were physical function, fatigue and appetite loss. Patient-reported physical function also had higher predictive performance compared to physiciandefined ECOG-PS.

PROs are an important measure that allow for the assessment of the patient's quality of life and has been increasingly utilized in clinical trials as an endpoint (3,79,11,19-21). However, their role in clinical practice has yet to be established. There is emerging data that their role can enhance decision making, improve patient satisfaction and be and inexpensive predictive and prognostic marker for survival $(3,7,9,11,21-26)$.

Our study reported a statistically significant association with OS for the pre-treatment PRO's physical function, fatigue, appetite loss, pain, role function, global health status, social function, dyspnoea, constipation, nausea and vomiting, insomnia, emotional function, cognitive function, and financial difficulty. These results are consistent with other studies investigating associations between pretreatment PROs and survival. Our study is the first to report an OS difference for the PROs of financial difficulty in mNSCLC patients. The association of increasing financial burden and a decrease in PFS was reported in one prior study however a statistically significant result was not seen for OS (27).

The pre-treatment PROs for physical function had the highest predictive performance for OS when compared to other PROs in our study. Other studies have also reported physical function to be highly associated with survival for advanced lung cancer patients, with some reporting for every 10-point decrease prior to treatment in The EORTC QLQ-C30 for physical function being associated with a 7\% to $8 \%$ increase risk of death $(4,5,9,20)$.

ECOG-PS is a physician assessment that serves as a surrogate for physical function (28). ECOG-PS is a crucial tool in treatment decision making and is widely utilized in clinical practice and randomized clinical trials to determine eligibility for treatment (23). ECOG-PS has five subcategories; 0 for patients with normal activity, 1 for patients with restrictions in strenuous activity but ambulatory and able to carry out light housework, 2 for patients who are ambulatory but unable to carry out housework, 3 for patients who are capable of only limited self-care and confined to a bed or chair for more than $50 \%$ of the waking hours, 4 for patients who are bed bound and unable to carry out self-care and 5 for a patient who is dead (28). ECOG-PS 0 to 1 has shown to be a positive prognostic and predictive marker of survival, a similar finding to our study (23).

However, ECOG-PS does have its limitations as studies have shown inaccuracy of the reporting with $40 \%$ to $50 \%$ of physician overestimating the patients performance status $(28,29)$. A discordance was also demonstrated between patient-reported physical function and physiciandefined ECOG-PS in our study. Highlighting the need to add PROs to improve our methods of assessing patients' performance status and function.

In our study, both patient-reported physical function and physician-defined ECOG-PS were independently prognostic of survival, with the former having a slightly higher predictive performance value. A similar study also reported physical function to be more predictive than ECOG-PS in NSCLC patients treated with atezolizumab monotherapy (26). This growing evidence demonstrates the potential complimentary role of implementing PROs into clinical practice (7,9,30-32). However, the integration of PROs into clinical practice poses challenges including time constraints, handling of missing data and literacy differences between patients. To circumvent these challenges, a more targeted approach of asking 
questions that make up patient-reported physical function such as 'Do you have any trouble doing strenuous activities?' or 'Do you have any trouble taking a short/long walk?' could be utilized in conjunction with ECOG-PS.

Our study is a post hoc analysis of phase III clinical trials thus providing high quality data for PROs, OS and PFS with minimal missing data. As there is a stringent criteria for enrolment into clinical trials, the generalisability of the results are impacted. For example, IMpower130, IMpower131 and IMpower150 did not include patients with ECOG-PS2 or above, which account for up to $25 \%$ of patients with mNCSLC in real world studies (33). In addition, patients aged above 75 are underrepresented in the clinical trials, representing about $10 \%$ of the cohort, thus further research is required to assess the generalizability of the results in this population group. It is also acknowledged that the EORTC-QLQ-C30 was developed in the era prior to the use of immunotherapies. It will be of interest to future research whether validated PROs tools specific for immunotherapies emerge, and if they do whether they have an improved ability for pre-treatment $\mathrm{PRO}$ reports to predict likely survival outcomes.

\section{Conclusions}

In patients with advanced NSCLC who received first line atezolizumab based therapy, pre-treatment PRO's were prognostic for survival outcomes. Patient-reported physical function had higher predictive performance compared to physician-defined ECOG-PS. These results suggest PRO can further be used in clinical practice as a stratification factor.

\section{Acknowledgments}

Funding: This work was supported by Australia's National Health and Medical Research Council (No. APP2008119) and Cancer Council South Australia.

\section{Footnote}

Reporting Checklist: The authors have completed the REMARK reporting checklist. Available at https://tlcr. amegroups.com/article/view/10.21037/tlcr-21-938/rc

Data Sharing Statement: Available at https://tlcr.amegroups. com/article/view/10.21037/tlcr-21-938/dss
Peer Review File: Available at https://tlcr.amegroups.com/ article/view/10.21037/tlcr-21-938/prf

Conflicts of Interest: All authors have completed the ICMJE uniform disclosure form (available at https://tlcr.amegroups. com/article/view/10.21037/tlcr-21-938/coif). RAM is supported by the Cancer Council South Australia; a board member of not-for-profit entity Therapeutic Innovation Australia. MJS reports grants from Cancer Council of South Australia, during the conduct of the study; grants from Pfizer, outside the submitted work. AMH is supported by an Investigator Grant from Australia's National Health and Medical Research Council. The other authors have no conflicts of interest to declare.

Ethical Statement: The authors are accountable for all aspects of the work in ensuring that questions related to the accuracy or integrity of any part of the work are appropriately investigated and resolved. The study was conducted in accordance with the Declaration of Helsinki (as revised in 2013). Secondary analysis of anonymized clinical trial data was confirmed negligible risk research by the Southern Adelaide Local Health Network, Office for Research and Ethics and was exempt from review as the project utilised publicly available de-identified data.

Open Access Statement: This is an Open Access article distributed in accordance with the Creative Commons Attribution-NonCommercial-NoDerivs 4.0 International License (CC BY-NC-ND 4.0), which permits the noncommercial replication and distribution of the article with the strict proviso that no changes or edits are made and the original work is properly cited (including links to both the formal publication through the relevant DOI and the license). See: https://creativecommons.org/licenses/by-nc-nd/4.0/.

\section{References}

1. Hanna NH, Schneider BJ, Temin S, et al. Therapy for Stage IV Non-Small-Cell Lung Cancer Without Driver Alterations: ASCO and $\mathrm{OH}(\mathrm{CCO})$ Joint Guideline Update. J Clin Oncol 2020;38:1608-32.

2. Aaronson NK, Ahmedzai S, Bergman B, et al. The European Organization for Research and Treatment of Cancer QLQ-C30: a quality-of-life instrument for use in international clinical trials in oncology. J Natl Cancer Inst $1993 ; 85: 365-76$. 
3. Vodicka E, Kim K, Devine EB, et al. Inclusion of patientreported outcome measures in registered clinical trials: Evidence from ClinicalTrials.gov (2007-2013). Contemp Clin Trials 2015;43:1-9.

4. Gupta D, Braun DP, Staren ED. Association between changes in quality of life scores and survival in nonsmall cell lung cancer patients. Eur J Cancer Care (Engl) 2012;21:614-22.

5. Ediebah DE, Coens C, Zikos E, et al. Does change in health-related quality of life score predict survival? Analysis of EORTC 08975 lung cancer trial. Br J Cancer 2014;110:2427-33.

6. Gotay CC, Kawamoto CT, Bottomley A, et al. The prognostic significance of patient-reported outcomes in cancer clinical trials. J Clin Oncol 2008;26:1355-63.

7. Mierzynska J, Piccinin C, Pe M, et al. Prognostic value of patient-reported outcomes from international randomised clinical trials on cancer: a systematic review. Lancet Oncol 2019;20:e685-98.

8. Quinten C, Coens C, Mauer M, et al. Baseline quality of life as a prognostic indicator of survival: a meta-analysis of individual patient data from EORTC clinical trials. Lancet Oncol 2009; 10:865-71.

9. Kerrigan K, Patel SB, Haaland B, et al. Prognostic Significance of Patient-Reported Outcomes in Cancer. JCO Oncol Pract 2020;16:e313-23.

10. Seow H, Tanuseputro P, Barbera L, et al. Development and Validation of a Prognostic Survival Model With Patient-Reported Outcomes for Patients With Cancer. JAMA Netw Open 2020;3:e201768.

11. Trejo MJ, Bell ML, Dhillon HM, et al. Baseline quality of life is associated with survival among people with advanced lung cancer. J Psychosoc Oncol 2020;38:635-41.

12. Jotte R, Cappuzzo F, Vynnychenko I, et al. Atezolizumab in Combination With Carboplatin and Nab-Paclitaxel in Advanced Squamous NSCLC (IMpower131): Results From a Randomized Phase III Trial. J Thorac Oncol 2020;15:1351-60.

13. West H, McCleod M, Hussein M, et al. Atezolizumab in combination with carboplatin plus nab-paclitaxel chemotherapy compared with chemotherapy alone as firstline treatment for metastatic non-squamous non-smallcell lung cancer (IMpower130): a multicentre, randomised, open-label, phase 3 trial. Lancet Oncol 2019;20:924-37.

14. Herbst RS, Giaccone G, de Marinis F, et al. Atezolizumab for First-Line Treatment of PD-L1-Selected Patients with NSCLC. N Engl J Med 2020;383:1328-39.

15. Bergman B, Aaronson NK, Ahmedzai S, et al. The
EORTC QLQ-LC13: a modular supplement to the EORTC Core Quality of Life Questionnaire (QLQ-C30) for use in lung cancer clinical trials. EORTC Study Group on Quality of Life. Eur J Cancer 1994;30A:635-42.

16. Fayers P, Bottomley A; EORTC Quality of Life Group, et al. Quality of life research within the EORTC-the EORTC QLQ-C30. European Organisation for Research and Treatment of Cancer. Eur J Cancer 2002;38 Suppl 4:S125-33.

17. Scott NW, Fayers PM, Aaronson NK, et al. EORTC QLQ-C30 Scoring Manual. Brussels: The European Organization for Research and Treatment of Cancer, 2001.

18. Scott NW, Fayers P, Aaronson NK, et al. EORTC QLQ-C30 reference values manual. 2008. Available online: https://abdn.pure.elsevier.com/en/publications/ eortc-qlq-c30-reference-values-manual

19. Sanders C, Egger M, Donovan J, et al. Reporting on quality of life in randomised controlled trials: bibliographic study. BMJ 1998;317:1191-4.

20. Braun DP, Gupta D, Staren ED. Quality of life assessment as a predictor of survival in non-small cell lung cancer. BMC Cancer 2011;11:353.

21. Steffen McLouth LE, Lycan TW Jr, Levine BJ, et al. Patient-Reported Outcomes From Patients Receiving Immunotherapy or Chemoimmunotherapy for Metastatic Non-Small-Cell Lung Cancer in Clinical Practice. Clin Lung Cancer 2020;21:255-263.e4.

22. Atkinson TM, Rogak LJ, Heon N, et al. Exploring differences in adverse symptom event grading thresholds between clinicians and patients in the clinical trial setting. J Cancer Res Clin Oncol 2017;143:735-43.

23. Bersanelli M, Brighenti $M$, Buti S, et al. Patient performance status and cancer immunotherapy efficacy: a meta-analysis. Med Oncol 2018;35:132.

24. Hall ET, Singhal S, Dickerson J, et al. Patient-Reported Outcomes for Cancer Patients Receiving Checkpoint Inhibitors: Opportunities for Palliative Care-A Systematic Review. J Pain Symptom Manage 2019;58:137-156.e1.

25. Nishijima TF, Shachar SS, Muss HB, et al. PatientReported Outcomes with PD-1/PD-L1 Inhibitors for Advanced Cancer: A Meta-Analysis. Oncologist 2019;24:e565-73.

26. Hopkins AM, Wagner J, Kichenadasse G, et al. Patientreported outcomes as a prognostic marker of survival in patients with advanced nonsmall cell lung cancer treated with immunotherapy. Int J Cancer 2020;147:3085-9.

27. Klein J, Bodner W, Garg M, et al. Pretreatment financial toxicity predicts progression-free survival following 
concurrent chemoradiotherapy for locally advanced nonsmall-cell lung cancer. Future Oncol 2019;15:1697-705.

28. Sørensen JB, Klee M, Palshof T, et al. Performance status assessment in cancer patients. An inter-observer variability study. Br J Cancer 1993;67:773-5.

29. Ando M, Ando Y, Hasegawa Y, et al. Prognostic value of performance status assessed by patients themselves, nurses, and oncologists in advanced non-small cell lung cancer. $\mathrm{Br}$ J Cancer 2001;85:1634-9.

30. Badaoui S, Kichenadasse G, Rowland A, et al. PatientReported Outcomes Predict Progression-Free Survival of Patients with Advanced Breast Cancer Treated with Abemaciclib. Oncologist 2021;26:562-8.

Cite this article as: Badaoui $\mathrm{S}$, Shahnam A, McKinnon RA, Abuhelwa AY, Sorich MJ, Hopkins AM. The predictive utility of patient-reported outcomes and performance status for survival in metastatic lung cancer patients treated with chemoimmunotherapy. Transl Lung Cancer Res 2022;11(3):432-439. doi: 10.21037/tlcr-21-938
31. Abuhelwa AY, Kichenadasse G, McKinnon RA, et al. Machine Learning for Prediction of Survival Outcomes with Immune-Checkpoint Inhibitors in Urothelial Cancer. Cancers (Basel) 2021;13:2001.

32. Hopkins AM, Kichenadasse G, Garrett-Mayer E, et al. Development and Validation of a Prognostic Model for Patients with Advanced Lung Cancer Treated with the Immune Checkpoint Inhibitor Atezolizumab. Clin Cancer Res 2020;26:3280-6.

33. Ivanović M, Knez L, Herzog A, et al. Immunotherapy for Metastatic Non-Small Cell Lung Cancer: Real-World Data from an Academic Central and Eastern European Center. Oncologist 2021;26:e2143-50. 
Table S1 Summary of patient characteristics by treatment arm

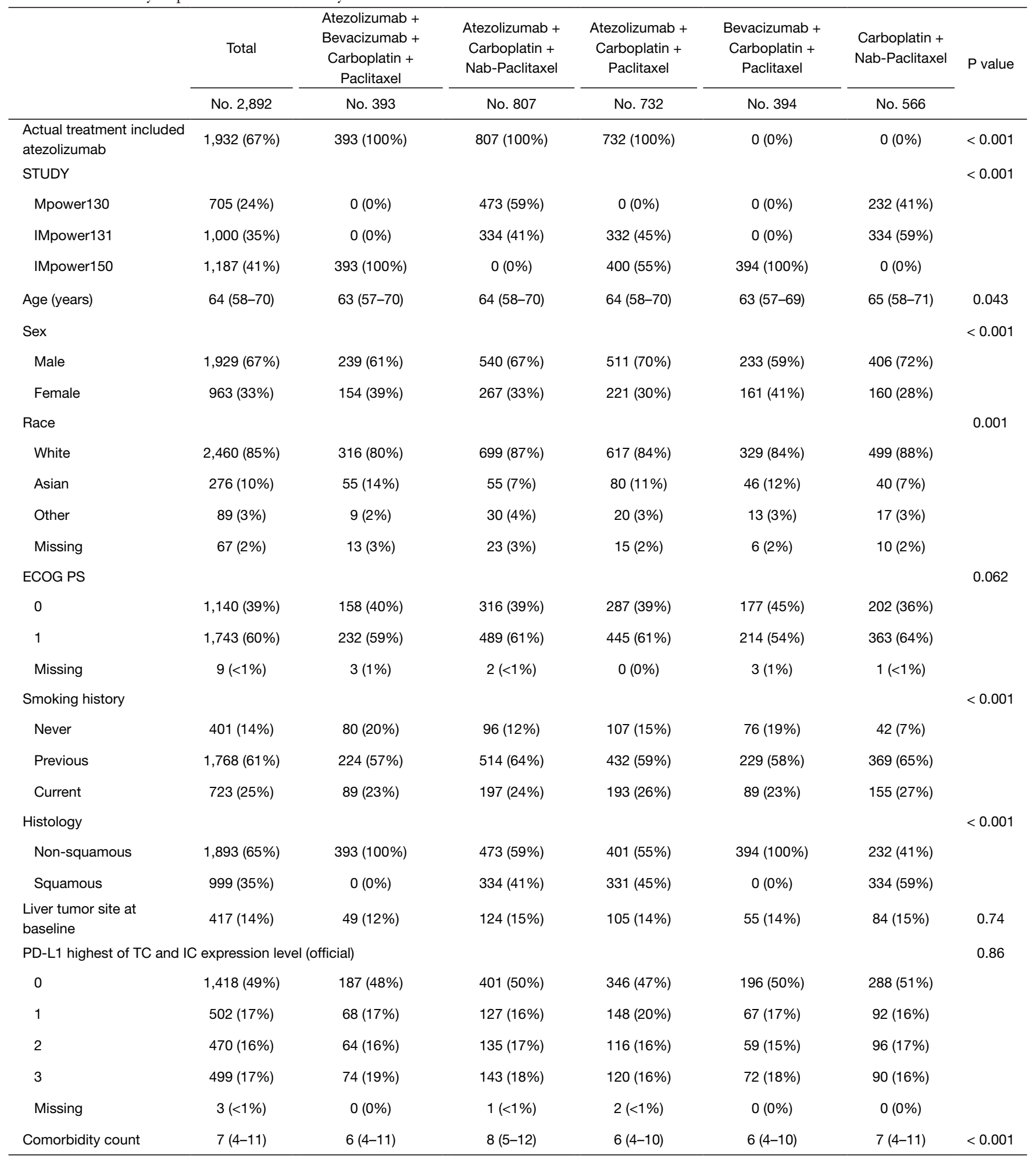

Data are median (IQR) or number of patients (\%). P values per Chi-Square test for categorical data and Kruskal-Wallis test for continuous data. 
Table S2 Summary of pre-treatment patient-reported outcome values by treatment arm

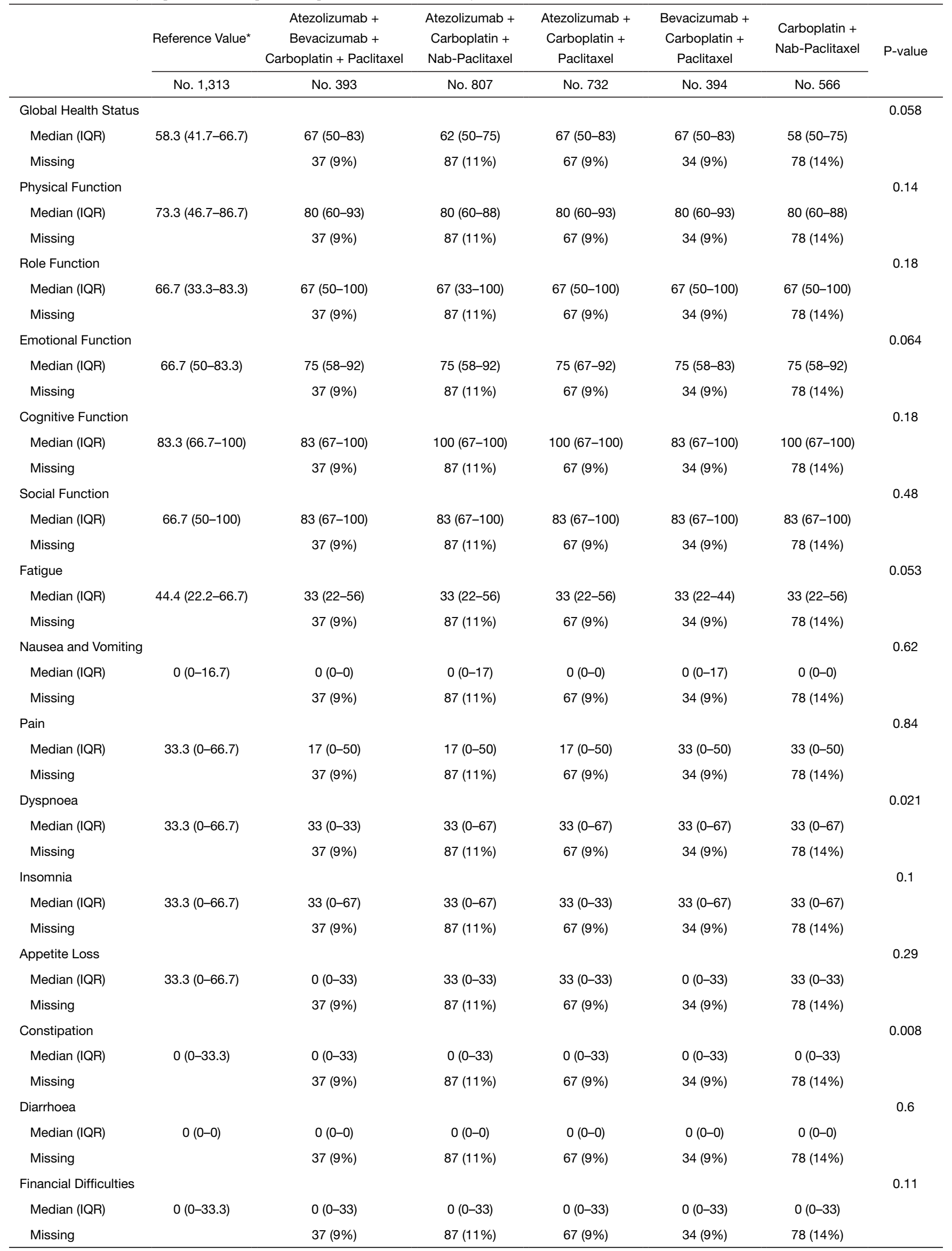

Data are median (IQR) or number of patients (\%). P values per Chi-Square test for categorical data and Kruskal-Wallis test for continuous data. *, Reference value according to EORTC QLQ-C30 Tables of Reference Values for Lung cancer: stage III-IV (p. 181). 
Table S3 Univariable and multivariable association between PROs and OS for patients treated with atezolizumab

\begin{tabular}{|c|c|c|c|c|c|c|c|c|c|}
\hline PRO & \multicolumn{5}{|c|}{ Univariable } & \multicolumn{4}{|c|}{ Multivariable ${ }^{\#}$} \\
\hline Physical Function & 1741 & 0.84 & 0.82 to 0.87 & $<0.001$ & 0.62 & 1686 & 0.87 & 0.84 to 0.89 & $<0.001$ \\
\hline Fatigue & 1741 & 1.15 & 1.12 to 1.18 & $<0.001$ & 0.61 & 1686 & 1.14 & 1.11 to 1.17 & $<0.001$ \\
\hline Appetite Loss & 1741 & 1.11 & 1.09 to 1.13 & $<0.001$ & 0.6 & 1686 & 1.1 & 1.08 to 1.12 & $<0.001$ \\
\hline Role Function & 1741 & 0.91 & 0.89 to 0.93 & $<0.001$ & 0.6 & 1686 & 0.93 & 0.91 to 0.95 & $<0.001$ \\
\hline Global Health Status & 1741 & 0.88 & 0.86 to 0.91 & $<0.001$ & 0.59 & 1686 & 0.9 & 0.87 to 0.93 & $<0.001$ \\
\hline Social Function & 1741 & 0.91 & 0.89 to 0.93 & $<0.001$ & 0.58 & 1686 & 0.92 & 0.89 to 0.94 & $<0.001$ \\
\hline Dyspnoea & 1741 & 1.07 & 1.05 to 1.09 & $<0.001$ & 0.56 & 1686 & 1.06 & 1.04 to 1.09 & $<0.001$ \\
\hline Insomnia & 1741 & 1.05 & 1.03 to 1.07 & $<0.001$ & 0.54 & 1686 & 1.04 & 1.02 to 1.07 & $<0.001$ \\
\hline Emotional Function & 1741 & 0.95 & 0.92 to 0.98 & $<0.001$ & 0.54 & 1686 & 0.95 & 0.92 to 0.98 & $<0.001$ \\
\hline Cognitive Function & 1741 & 0.95 & 0.92 to 0.98 & 0.002 & 0.52 & 1686 & 0.96 & 0.93 to 0.99 & 0.013 \\
\hline Financial Difficulties & 1741 & 1.03 & 1.01 to 1.05 & 0.009 & 0.52 & 1686 & 1.03 & 1.00 to 1.05 & 0.035 \\
\hline Diarrhoea & 1741 & 1 & 0.96 to 1.04 & 0.997 & 0.5 & 1686 & 1 & 0.95 to 1.04 & 0.851 \\
\hline
\end{tabular}

$\mathrm{Cl}$, confidence interval; HR, hazard ratio, *, HR based on 10-unit increase, ", Adjusted for Age, Sex, Race, ECOG-PS, Smoking history, Histology, Liver tumor site at baseline, PD-L1 highest of TC and IC expression level (official) and comorbidity count. 


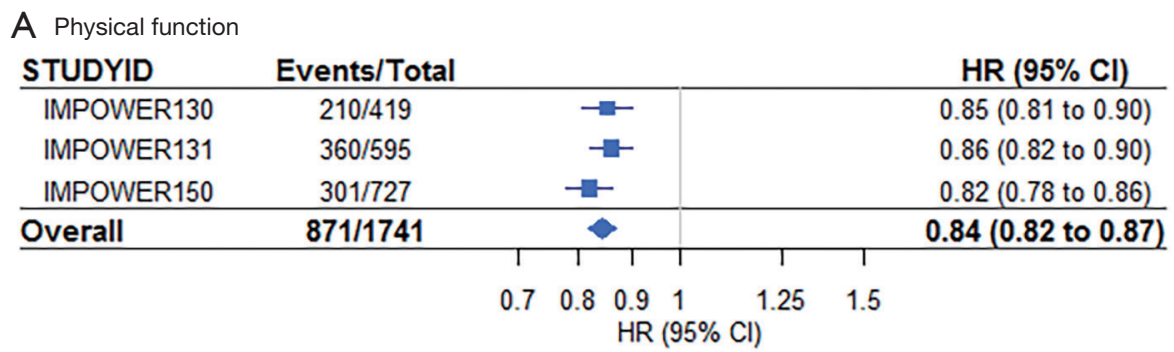

B Fatigue

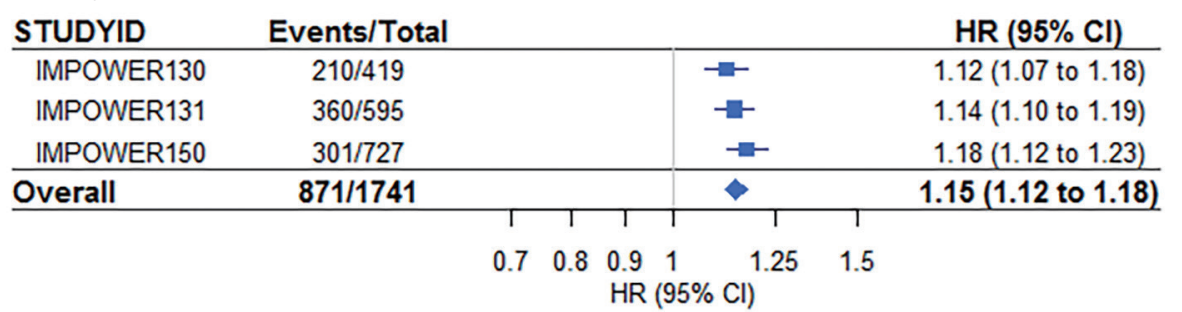

C Fatigue

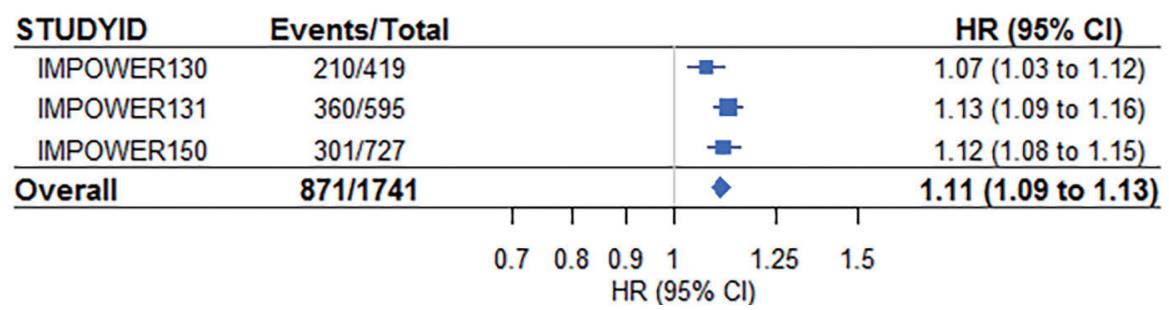

Figure S1 Univariable association between patient-reported physical function, fatigue and appetite loss with OS for patients treated with atezolizumab by study.

Table S4 Univariable and multivariable association between PROs and PFS for patients treated with atezolizumab

\begin{tabular}{|c|c|c|c|c|c|c|c|c|c|}
\hline \multirow[t]{2}{*}{ PRO } & \multicolumn{5}{|c|}{ Univariable } & \multicolumn{4}{|c|}{ Multivariable $^{\#}$} \\
\hline & $\mathrm{n}$ & $\mathrm{HR}^{*}$ & $95 \% \mathrm{Cl}$ & $\mathrm{P}$ & c & $\mathrm{n}$ & $\mathrm{HR}^{*}$ & $95 \% \mathrm{Cl}$ & $\mathrm{P}$ \\
\hline Fatigue & 1741 & 1.09 & 1.07 to 1.11 & $<0.001$ & 0.57 & 1686 & 1.08 & 1.06 to 1.11 & $<0.001$ \\
\hline Pain & 1741 & 1.08 & 1.06 to 1.10 & $<0.001$ & 0.57 & 1686 & 1.07 & 1.05 to 1.09 & $<0.001$ \\
\hline Role Function & 1741 & 0.94 & 0.92 to 0.95 & $<0.001$ & 0.56 & 1686 & 0.95 & 0.93 to 0.97 & $<0.001$ \\
\hline Appetite Loss & 1741 & 1.06 & 1.04 to 1.08 & $<0.001$ & 0.56 & 1686 & 1.05 & 1.04 to 1.07 & $<0.001$ \\
\hline Social Function & 1741 & 0.93 & 0.91 to 0.95 & $<0.001$ & 0.55 & 1686 & 0.94 & 0.92 to 0.96 & $<0.001$ \\
\hline Dyspnoea & 1741 & 1.04 & 1.02 to 1.06 & $<0.001$ & 0.54 & 1686 & 1.04 & 1.02 to 1.06 & $<0.001$ \\
\hline Emotional Function & 1741 & 0.95 & 0.93 to 0.97 & $<0.001$ & 0.53 & 1686 & 0.95 & 0.93 to 0.97 & $<0.001$ \\
\hline Constipation & 1741 & 1.05 & 1.03 to 1.07 & $<0.001$ & 0.53 & 1686 & 1.04 & 1.02 to 1.06 & $<0.001$ \\
\hline Financial Difficulties & 1741 & 1.02 & 1.00 to 1.04 & 0.044 & 0.51 & 1686 & 1.01 & 0.99 to 1.03 & 0.251 \\
\hline Diarrhoea & 1741 & 1.01 & 0.98 to 1.05 & 0.468 & 0.5 & 1686 & 1.01 & 0.98 to 1.05 & 0.565 \\
\hline
\end{tabular}

$\mathrm{Cl}=$ confidence interval, HR=hazard ratio, *, HR based on 10-unit increase, \#, Adjusted for Age, Sex, Race, ECOG-PS, Smoking history, Histology, Liver tumor site at baseline and PD-L1 highest of TC and IC expression level (official). 
Table S5 Summary of patient-reported physical function raw scores by physician- assessed ECOG-PS in patients treated with atezolizumab

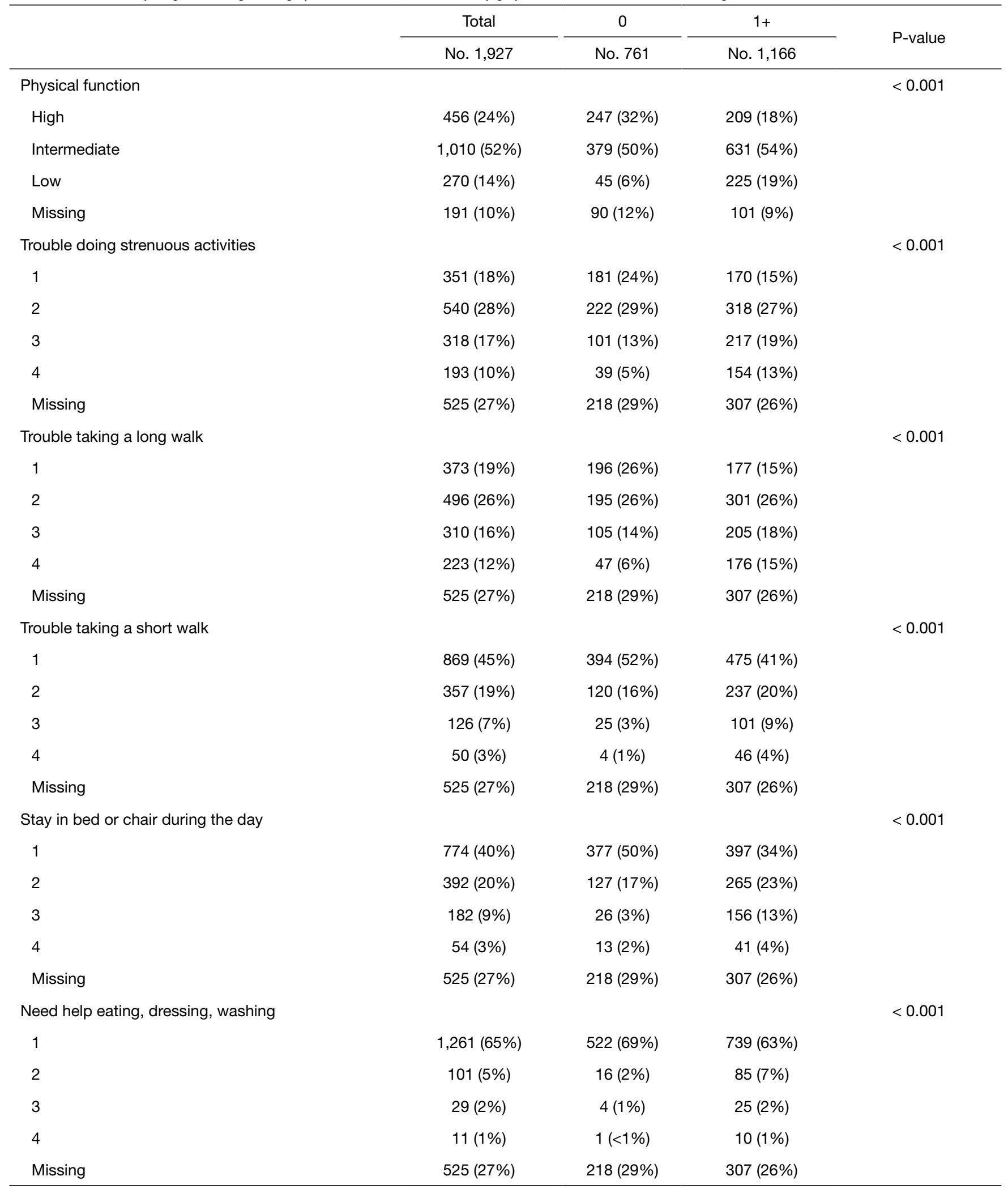

Data are median (IQR) or number of patients (\%). P values per Chi-Square test for categorical data and Kruskal-Wallis test for continuous data. $1=$ Not at All, $2=$ A Little, $3=$ Quite a bit, 4 = Very much. 
Table S6 Associations between patient-reported physical function and ECOG-PS with PFS for patients treated with atezolizumab

\begin{tabular}{|c|c|c|c|c|c|c|c|}
\hline & \multicolumn{4}{|c|}{ Univariable } & \multicolumn{3}{|c|}{ Multivariable $^{\#}$} \\
\hline Physical function & & & $<0.001$ & 0.56 & & & $<0.001$ \\
\hline Low & 270 & 1.86 [1.57 to 2.21$]$ & & & 270 & 1.71 [1.43 to 2.04$]$ & \\
\hline Intermediate & 1013 & 1.34 [1.18 to 1.53$]$ & & & 1010 & 1.30 [1.14 to 1.49$]$ & \\
\hline ECOG-PS & 1927 & & $<0.001$ & 0.55 & & & $<0.001$ \\
\hline 0 & 761 & 1 & & & 671 & & \\
\hline $1+$ & 1166 & 1.44 [1.30 to 1.60$]$ & & & 1065 & $1.32[1.17$ to 1.48$]$ & \\
\hline
\end{tabular}

\#, Model includes pre-treatment physical function and ECOG-PS. Cl, confidence interval; HR, hazard ratio. 